\title{
Die Europäische Kommission und ein Gemeinsames Europäisches Kaufrecht
}

\author{
von Hans Christoph Grigoleit
}

\section{Vorbemerkungen: Rahmenbedingungen des Verordnungs- Entwurfs für ein Gemeinsames Europäisches Kaufrecht (Draft Common European Sales Law, DCESL)}

Die EU-Kommission hat am 11. Oktober 2011 einen Verordnungs-Entwurf für ein Gemeinsames Europäisches Kaufrecht vorgelegt. Diese neue Kaufrechtsordnung wird als optionales Instrument bezeichnet, weil sie ihre Geltung nicht (allein) durch hoheitliche Anordnung erhalten soll, sondern (auch) durch die einvernehmliche Entscheidung der Vertragsparteien für ihre Einbeziehung (opt-in). Die neue Wahlordnung soll für transnationale Kaufgeschäfte im Binnenmarkt zur Verfügung stehen, und zwar sowohl für Verträge zwischen Unternehmen (business-to-business, b2b) als auch für solche zwischen Unternehmen und Verbrauchern (business-to-consumer, b2c). Den Mitgliedstaaten ist die Zulassung der Wahlordnung auch für innerstaatliche Transaktionen offen gelassen.

\section{Status quo des Angleichungsprozesses im Privatrecht: fragmentarische Eingriffe - Verbraucherschutzrecht, Anti- Diskriminierungsrecht, Verkehrsschutz}

Die Kommission schlägt mit dem Entwurf eine neue Richtung im Prozess der Privatrechtsangleichung ein, indem für den elementarsten Vertragstyp, den Kaufvertrag, ein breitflächiges Regelwerk geschaffen werden soll - gleichsam ein Kodex in Miniaturformat mit (durch den „Einwahlvorbehalt“) eingeschränkter Verbindlichkeit. Charakteristisch für die bisherigen auf das Privatrecht bezogenen Rechtssetzungsakte seitens der Union war demgegenüber die Konzentration auf fragmentarische Schutzinstrumente für Verbraucherverträge, namentlich auf die Verankerung von Widerrufsrechten, detaillierten Informationspflichten und einzelnen Festlegungen zwingenden Rechts. 
Diese Schutzinstrumente wurden durch zahlreiche Einzelrichtlinien implementiert; sie sind mit bestimmten Absatzformen (Fernabsatz einschl. des elektronischen Geschäftsverkehrs, Haustürgeschäfte) und Vertragstypen (Verbrauchsgüterkauf, Verbraucherkredit, Pauschalreiseverträge und Teilzeitwohnrechteverträge) verknüpft. Eine eigene Kategorie bildet die Klauselkontrolle, die im Hinblick auf alle Verbraucherverträge eine Inhaltskontrolle für vorformulierte Klauseln vorsieht. Die im Jahr 2011 verabschiedete Verbraucherrechterichtlinie (Richtlinie 2011/83/EU, Umsetzung bis 2013) konsolidiert und reformiert im Wesentlichen den absatzbezogenen Verbraucherschutz. Die wichtigsten Ansätze für eine privatrechtliche Angleichung außerhalb des Verbraucherschutzrechts bilden die Anti-Diskriminierungs-Richtlinien und einzelne elementare Verkehrsschutzregelungen, namentlich zur Absicherung des Zahlungsverkehrs (Überweisungs-, Zahlungsverzugsrichtlinie).

\section{Ansätze zu einem weitergreifenden Kodifikationsprozess: akademische Modellregeln und das Projekt des gemeinsamen Referenzrahmens}

Eine Überwindung des fragmentarischen Charakters der Privatrechtsangleichung wird seit langem von akademischen Arbeitsgruppen vorbereitet, die mit Unterstützung der EU verschiedene Modellgesetze erarbeitet und vorgelegt haben. Zu nennen sind in diesem Zusammenhang zunächst die von der sog. Lando-Gruppe konzipierten Principles of European Contract Law, die im Wege einer kritischrechtsvergleichenden Methode entwickelt wurden und Regelungen für das allgemeine Vertragsrecht enthalten. Die sog. Study-Group, die als Nachfolgeorganisation der Lando-Gruppe gelten darf, hat weitere Modellentwürfe insbesondere für verschiedene Vertragstypen sowie für gesetzliche Schuldverhältnisse konzipiert. Schließlich hat die sog. Acquis-Gruppe den im Privatrecht bereits vorhandenen (soeben skizzierten) EU-Acquis durch Systematisierung, Generalisierung und Ergänzung zu einem kodexartigen Regelwerk weiterentwickelt.

Der bislang weitreichendste akademische Modellentwurf ist der sog. Draft Common Frame of Reference (DCFR, 2009), der Entwurf eines gemeinsamen Referenzrahmens. Er führt im Wesentlichen die Regelwerke der Lando-Gruppe, der Study-Group und der Acquis-Gruppe zusammen und behandelt das allgemeine Vertragsrecht, das Vertragstypenrecht, das Verbraucherschutzrecht, ein rudimentäres Mobiliarsachenrecht und verschiedene gesetzliche Schuldverhältnisse. Der Entwurf des Referenzrahmens kommt sachlich sehr nahe an eine umfassende 
Kodifikation des Bürgerlichen Rechts heran; er erstreckt sich auf das gesamte Vermögensrecht und spart - aus der Perspektive des BGB - im Wesentlichen nur das Familien- und Erbrecht aus.

In den vergangenen Jahren hat die Kommission ihre Bereitschaft zu weiterer Rechtssetzung im Privatrecht in verschiedenen Verlautbarungen bekundet und dabei alternative Modelle zur Diskussion gestellt - zuletzt etwa im „Grünbuch Europäisches Vertragsrecht 2010“. Neben dem nunmehr in das Entwurfsstadium geführten Projekt eines optionalen Regelwerks stand für einige Zeit auch das von den akademischen Gruppen durch den erwähnten Entwurf (DCFR) vorbereitete - Projekt des Common Frame of Reference (CFR) im Fokus der Pläne der Kommission. Es war daran gedacht, dass der Referenzrahmen als toolbox, also als Hilfsmaterial und Inspirationsquelle für künftige Gesetzgebung auf Unionsebene oder auch auf der Ebene der Mitgliedstaaten, Verwendung finden könnte.

Allerdings ist es zweifelhaft geblieben, welche legitime, hinreichend rationalisierbare Funktion die politische Sanktionierung eines solchen soft law-Kodex durch EU-Organe erfüllen kann. Auch sind grundlegende Einwände gegen die Qualität des vorgelegten Entwurfs und dessen erhebliche Trag- und Reichweite erhoben worden. Wohl auch im Lichte dieser Erwägungen verfolgt die Kommission das Projekt des gemeinsamen Referenzrahmens gegenwärtig nicht weiter.

\section{Positionierung des DCESL im Prozess der europäischen Privat- rechtsangleichung}

Der Entwurf für ein CESL trägt den Einwänden gegen den DCFR vor allem dadurch Rechnung, dass der sachliche Anwendungsbereich und die Regelungsdichte stark reduziert werden (Begrenzung auf Kaufverträge und auch insoweit keine lückenlose Regelung). Das CESL teilt jedoch mit dem Projekt des Referenzrahmens die Qualität eines soft law-Instruments in dem Sinne, dass die EU kein unmittelbar geltendes Kaufrecht schafft - vermutlich um die Akzeptanz seitens der Mitgliedstaaten, der Verbände und der Fachöffentlichkeit zu erhöhen. Freilich erhält das DCESL vermittels der „Einwahl“ der Parteien durchaus rechtliche Verbindlichkeit, so dass es im Gegensatz zum DCFR rechtsquellentheoretisch eine klar definierbare Funktion erfüllt, aber auch den Geltungsanspruch der mitgliedstaatlichen Rechtsordnungen in stärkerem Maße in Frage stellt.

Inhaltlich übernimmt das CESL in seinem reduzierten Regelungsbereich vielfach Regelungen des DCFR bzw. der diesem zugrundegelegten akademischen Modellentwürfe. Die EU-Kommission hat eine Expertengruppe mit einer inhaltli- 
chen Reduktion und Überarbeitung (Commission Expert Group on European Contract Law) der Vorgaben des DCFR betraut, deren Arbeit sich in einen Vorentwurf (Mai 2011) und auch in der endgültigen Fassung des DCESL niedergeschlagen hat. Personell steht die Gruppe der beteiligten Experten in starker Kontinuität zu den am DCFR Beteiligten.

Im Einzelnen findet sich im DCESL - wie auch im DCFR - eine Kombination aus allgemeinen Regeln des Vertrags- bzw. Kaufrechts und aus Regeln des Verbrauchervertragsrechts. Die allgemeinen Vorschriften (insbesondere: Vertragsschluss, Irrtumsrecht, Auslegung, Vertragspflichten/Leistungsstörungsrecht) finden überwiegend keine Anknüpfung im geltenden europäischen Privatrecht, sie sind vielmehr in Anlehnung an die mitgliedstaatlichen Vertragsrechtsordnungen entwickelt worden. Die Regeln des Verbrauchervertragsrechts (insbesondere: Widerrufsrecht, Informationspflichten, Klauselkontrolle) sind aus den vorhandenen Richtlinien übernommen worden bzw. erweitern und verallgemeinern deren Ansätze. Erweiterungen des Anwendungsbereichs des vorhandenen Acquis ergeben sich etwa daraus, dass Informationspflichten und eine Klauselkontrolle auch für b2b-Verträge vorgesehen werden.

Der DCESL ist planmäßig lückenhaft, d.h. ihm ist nicht der Anspruch unterlegt, die in Frage stehenden Kaufverträge abschließend zu regeln. Vielmehr ist eine Ergänzung durch mitgliedstaatliches Recht hinsichtlich wesentlicher Regelungsbereiche erforderlich. Auf mitgliedstaatliches Recht wird etwa verwiesen hinsichtlich der vertraglichen Inhaltskontrolle aufgrund von Gesetzes- und Sittenwidrigkeit, der Diskriminierungsverbote, der Stellvertretung, des Parteiwechsels (Abtretung, Schuldübernahme etc.), des Sachenrechts und einer begleitenden gesetzlichen Schadensersatzhaftung (z.B. Produzentenhaftung).

Bekanntlich findet sich in den EU-Verträgen keine sachgebietspezifische EUKompetenz für das Privat-, Vertrags-, Kauf- oder auch nur Verbraucherschutzrecht. Die Kommission stützt den Verordnungs-Entwurf daher auf die Querschnittskompetenz des Art. 114 AEUV. Demgemäß wird von der Kommission der Gesichtspunkt der Binnenmarktförderung als zentrales Anliegen des DCESL formuliert: Der Handel zwischen den Mitgliedstaaten soll dadurch gefördert werden, dass vermittels des DCESL Rechtssicherheit für Unternehmen und Verbraucher bei grenzüberschreitenden Geschäften hergestellt wird. 


\section{Bezug der Stellungnahme}

Der Rechtsausschuss des Deutschen Bundestags hat am 21.11.2011 eine öffentliche Anhörung zum DCESL abgehalten, die insbesondere zur Vorbereitung der Entscheidung über die Erhebung der Subsidiaritätsrüge dienen sollte. Anlässlich dieser Anhörung wurde der Autor um eine Stellungnahme gebeten, die - in ihrer schriftlichen Fassung, ergänzt durch mündlich vorgetragene Erwägungen - die Basis dieses Berichts bildet. Der Stellungnahme ist im Hinblick auf ihre Funktion kein „Fußnotenapparat" beigefügt; ihre wissenschaftliche Grundlage bildet eine Reihe unterschiedlicher Studien. ${ }^{1}$ Inzwischen hat der Bundestag in Befolgung einer Empfehlung des Rechtsausschusses aufgrund seines Beschlusses vom 1. Dezember 2011 Subsidiaritätsrüge erhoben. ${ }^{2}$

\section{VI. Öffentliche Anhörung des Bundestags-Rechtsausschusses am 21.11.2011 - Vorschlag der EU-Kommission für ein Gemeinsa- mes Europäisches Kaufrecht - KOM(2011)635 - KOM(2011) 636: Stellungnahme}

Auf Anfrage des Rechtsausschusses des Deutschen Bundestags wurde zum Vorschlag der EU-Kommission für eine Verordnung über ein Gemeinsames Europäisches Kaufrecht wie folgt Stellung genommen:

Der Vorschlag der EU-Kommission für eine Verordnung über ein Gemeinsames Europäisches Kaufrecht verdient keine Unterstützung. Der Entwurf leidet an gravierenden konzeptionellen Defiziten. Aus Sicht des Deutschen Bundestags fällt besonders ins Gewicht, dass die EU-Verträge für eine derartige Gesetzgebungsinitiative keine Kompetenz enthalten.

\section{Wesentliche Feststellungen}

a) Fehlen der institutionellen Rahmenbedingungen und das Problem der Rechtsunsicherheit. Die institutionellen Rahmenbedingungen für die Schaffung einer verlässlichen Europäischen Kaufrechtsordnung sind nicht vorhanden. Insbesondere fehlt es auf europäischer Ebene an einer einheitlichen Zivilgerichts-

1 Vgl. insbesondere Eidenmüller/Faust/Grigoleit/Jansen/Wagner/Zimmermann: Der Gemeinsame Referenzrahmen für das Europäische Privatrecht, JZ 2008, 529 ff.; Grigoleit: Der Verbraucheracquis und die Entwicklung des Europäischen Privatrechts, AcP 210 (2010), 354 ff.; Eidenmüller/Faust/Grigoleit/Jansen/Wagner/Zimmermann: Revision des Verbraucher-acquis, 2011.

2 Zur Beschlussempfehlung und zum Bericht des Rechtsausschusses vgl. BT-Drucks. 17/8000. 
barkeit und an einer Verständigung über elementare Grundsätze der privatrechtlichen Regelbildung. Ohne diese Rahmenbedingungen schafft eine neue Kaufrechtsordnung keine Rechtssicherheit, sondern zusätzliche Rechtsunsicherheit.

b) Inhaltliche Mängel und Lückenhaftigkeit des Entwurfs. Der Entwurf für ein Europäisches Kaufrecht beruht auf unausgereiften akademischen Modellgesetzen. Er enthält eine Vielzahl einschneidender Regeln, die inkonsistent sind bzw. zu massiven Brüchen mit dem geltenden Vertragsrecht führen. Außerdem ist der Entwurf planmäßig lückenhaft; seine Anwendung ist in elementaren Aspekten auf eine ergänzende Heranziehung nationalen Rechts angewiesen. Auch deswegen ist es ausgeschlossen, dass er zur Stärkung der Rechtssicherheit beitragen kann.

c) Mangelnde Attraktivität der Wahlordnung für die Parteien im Binnenmarkt. Weder für Unternehmer noch für Verbraucher bringt das Europäische Kaufrecht einen erkennbaren Nutzen. Es ist nicht zu erwarten, dass das Europäische Kaufrecht eine nennenswerte Akzeptanz finden wird. Erst recht ist nicht anzunehmen, dass Binnenmarkttransaktionen durch eine Europäische Kaufrechtsordnung erleichtert werden: Verglichen mit den Belastungen, die sich aus der Sprachenvielfalt und den Herausforderungen einer Rechtsberatung bzw. einer gerichtlichen Rechtsdurchsetzung im Ausland ergeben, spielt die Varianz der Vertragsrechtsordnungen ohnehin eine verhältnismäßig geringe Rolle.

d) Beeinträchtigung des Verbraucherschutzes: Wegfall des Mindeststandards der Heimatrechtsordnung. Nach Art. 6 II der Rom-I-VO ist den Verbrauchern bei Geschäften im Binnenmarkt weitreichend der (vertraute) Schutzstandard ihrer Heimatrechtsordnung garantiert. Der Entwurf steht in einem unauflöslichen Wertungswiderspruch zu dieser Schutzgewährleistung.

e) Keine Neutralisierung der Einwände durch die optionale Ausgestaltung (,Unschädlichkeits-Hypothese“). Das Wahlrecht der Vertragsparteien neutralisiert die Einwände gegen den Entwurf nicht: Ein optionales Instrument verleiht den unausgereiften akademischen Regeln Autorität und führt zu voreiligen Festlegungen im Hinblick auf spätere verbindliche Vereinheitlichungsmaßnahmen. Eine rationale Entscheidung der Parteien über die Qualität des Entwurfs ist nicht möglich, weil sich dessen konkrete Auswirkungen nicht hinreichend klar bestimmen lassen: Ohne die Ausdifferenzierungen des Richterrechts entscheiden sich die Parteien für die „Katze im Sack“.

f) Kompetenzdefizit. Es ist weitgehend anerkannt, dass es für ein Vertragsgesetzbuch keine belastbare EU-Kompetenz gibt. Die optionale Ausgestaltung des 
Europäischen Kaufrechts behebt dieses Kompetenzdefizit ebenso wenig wie die Beschränkung des Anwendungsbereichs auf Binnenmarkt-Transaktionen: Denn im Ergebnis bleibt es dabei, dass durch das Europäische Kaufrecht mitgliedstaatliches Recht außer Kraft gesetzt wird. Es ist - auch angesichts der allgemeinen Probleme einer Rechtsdurchsetzung im Ausland - nicht plausibel, dass der Binnenmarkt durch den Entwurf eines Europäischen Kaufrechts in spürbarer Weise gefördert wird (Art. 114 AEUV). Das optionale Instrument würde vielmehr der schleichenden EU-Kompetenzanmaßung weiteren Vorschub leisten.

\section{Rechtliche und rechtspolitische Würdigung}

a) Fehlen der institutionellen Rahmenbedingungen und das Problem der Rechtsunsicherheit. Die institutionellen Rahmenbedingungen für die Schaffung einer verlässlichen Europäischen Kaufrechtsordnung sind nicht vorhanden. Insbesondere fehlt es auf europäischer Ebene an einer einheitlichen Zivilgerichtsbarkeit und an einer Verständigung über elementare Grundsätze der privatrechtlichen Regelbildung. Ohne diese Rahmenbedingungen schafft eine neue Kaufrechtsordnung keine Rechtssicherheit, sondern zusätzliche Rechtsunsicherheit.

aa) Im Kaufrecht (bzw. allgemein im Vertragsrecht) stammt die ganz überwiegende Zahl der praktizierten Regeln aus dem Richterrecht. Ein europäisches Vertragsgesetz (oder optionales Instrument) legt daher nur einen Bruchteil der benötigten Regeln fest; die übrigen Vorschriften müssen in einem jahrzehntelangen Prozess von den europäischen Gerichten entwickelt werden.

ab) Es gibt in Europa keine einheitliche Zivilgerichtsbarkeit. Ohne einheitliche Zivilgerichtsbarkeit kann kein einheitliches europäisches Kaufrecht entwickelt werden. Der EuGH ist zur Sicherung breitflächiger Rechtseinheit im Vertragsrecht weder kompetenziell noch organisatorisch in der Lage.

ac) Die europäischen Vertragsrechtsordnungen unterscheiden sich deutlich im Hinblick auf grundlegende Wertungen (Liberalität, Sozialschutz, Formalisierungsgrad etc.) und die Methodik der Regelbildung (etwa: englisches Common Law vs. kontinentaleuropäische Kodifikationen). Ohne eine Annäherung der Wertungsgrundlagen und Methoden können die nationalen Gerichte ein neues Kaufrecht nicht verlässlich zur Anwendung bringen.

b) Inhaltliche Mängel und Lückenhaftigkeit des Entwurfs. Der Entwurf für ein Europäisches Kaufrecht beruht auf unausgereiften akademischen Modellgesetzen. Er enthält eine Vielzahl einschneidender Regeln, die inkonsistent sind 
bzw. zu massiven Brüchen mit dem geltenden Vertragsrecht führen. Außerdem ist der Entwurf planmäßig lückenhaft; seine Anwendung ist in elementaren Aspekten auf eine ergänzende Heranziehung nationalen Rechts angewiesen. Auch deswegen ist es ausgeschlossen, dass er zur Stärkung der Rechtssicherheit beitragen kann.

ba) Der Entwurf beruht auf akademischen Modellgesetzen, die ihrerseits nicht ausgereift und weder in der Wissenschaft noch in der Praxis hinreichende Akzeptanz haben. Eine seriöse wissenschaftliche Prüfung und Diskussion hat noch nicht stattgefunden; angesichts der Komplexität und Bedeutung der Materie sollte diesem Prozess erhebliche Zeit gelassen werden.

bb) Der Entwurf enthält eine Vielzahl einschneidender Regelungen, die zu massiven Inkonsistenzen führen und sich erheblich von tradierten Wertungen des deutschen Rechts, aber auch der übrigen europäischen Privatrechtsordnungen entfernen. Bereits auf der Grundlage einer ersten Durchsicht lassen sich etwa ohne jeden Anspruch auf Vollständigkeit - folgende Einwände anführen:

- Der Entwurf regelt in vielfältiger Hinsicht den vorvertraglichen Kontakt (etwa Art. 13-39 DCESL). In dieser Phase wird (bislang üblicherweise) noch kein Vertrag geschlossen und damit auch die Wahl hinsichtlich des DCESL nicht ausgeübt (Rückwirkung? Aufgespaltene Rechtsgeltung?).

- Der Entwurf bringt unkalkulierbare Restriktionen der Vertragsfreiheit, insbesondere auf der Grundlage vager Generalklauseln, etwa Art. 2 Abs. 2 DCESL (Gebote von „Treu und Glauben“ als allgemeiner Schadensersatzanspruch) oder Art. 3 (allgemeine Kooperationspflicht).

- Der Entwurf vervielfältigt Informationspflichten in undurchsichtiger Weise und versieht diese mit weitreichenden Sanktionen und zwar sowohl in b2vwie auch b2b-Kontakten (Art. 13-29 DCESL).

- Der Entwurf enthält schwerwiegende Inkonsistenzen im Bereich des Irrtumsrechts (Art. 48 ff. DCESL). So läuft etwa die Definition des Irrtums auf eine redundante und verworrene Kombination des Verursacherprinzips und der Verantwortlichkeit für Pflichtverletzung hinaus (Art. 48 Abs. 1 (a), (b) iiv DCESL), das Problem der Drittverantwortlichkeit für Irrtum, Täuschung und Drohung ist nicht berücksichtigt (Art. 48-50 DCESL).

- Der Entwurf ist offen für einen Ausgleich immaterieller Schäden (,loss“ bzw „Verlust“, Art. 29, 55, 159 DCESL). Damit drohen den Parteien unabsehbare Haftungsfolgen.

- Das Recht des Verkäufers auf „Zweite Andienung“ im Fall mangelhafter Leistung (Nachbesserung, Neulieferung) wird durch den Entwurf weitgehend beseitigt (insbes. Art. 114, 115, 159 DCESL). Dies ist aus ökonomi- 
scher Perspektive nicht ratsam, weil dadurch u.U. ein bereits erbrachter Leistungsaufwand des Verkäufers entwertet wird.

- Die Verjährungsregeln des Entwurfs sind vage und lückenhaft. Das ist insbesondere deswegen bedenklich, weil im Bereich der Verjährung ein besonderes Bedürfnis nach Rechtssicherheit besteht. Es fehlt etwa eine klare Abgrenzung zwischen der „kurzen (2 J.) und der langen $\left(\begin{array}{lll}10 & \mathrm{~J}\end{array}\right)^{\text {“ }}$ Verjährungsfrist (Art. 179, 180 DCESL), ein deutlicher und einheitlicher Bezugspunkt für die Hemmung durch die „Einleitung gerichtlicher Verfahren" (Art. 181 DCESL) und eine unzweideutige Absicherung der dauerhaften Durchsetzbarkeit titulierter Ansprüche.

bc) Der Entwurf ist planmäßig lückenhaft und es ist daher in jedem Streitfall eine ergänzende Anwendung nationalen Vertragsrechts unverzichtbar. Nach nationalem Recht bleiben etwa so elementare Fragen zu behandeln, wie:

- Die vertragliche Inhaltskontrolle wegen Gesetzes- und Sittenwidrigkeit. Die Inhaltskontrolle nach den nationalen Sittenwidrigkeitsstandards konkurriert in unkalkulierbarer Weise mit der AGB-Kontrolle nach dem DCESL,

- die Diskriminierungsverbote,

- die Stellvertretung,

- der Parteiwechsel (Abtretung, Schuldübernahme etc.),

- das Sachenrecht, d.h. insbesondere Eigentumsfragen sowie

- die gesetzliche Schadensersatzhaftung, z.B. wegen unerlaubter Handlungen (insbes. Produzentenhaftung).

Dadurch wird in zentralen Punkten eine Abstimmung mit 27 Rechtsordnungen erforderlich. Das neue Kaufrecht schafft somit nur ein unkalkulierbares Potpourri aus europäischem und nationalem Recht, nicht aber effektive Rechtseinheit und schon gar keine Rechtssicherheit.

c) Mangelnde Attraktivität der Wahlordnung für die Parteien im Binnenmarkt. Weder für Unternehmer noch für Verbraucher bringt das Europäische Kaufrecht einen erkennbaren Nutzen. Es ist nicht zu erwarten, dass das Europäische Kaufrecht eine nennenswerte Akzeptanz finden wird. Erst recht ist nicht zu anzunehmen, dass Binnenmarkttransaktionen durch eine Europäische Kaufrechtsordnung erleichtert werden: Verglichen mit den Belastungen, die sich aus der Sprachenvielfalt und den Herausforderungen einer Rechtsberatung bzw. gerichtlichen Rechtsdurchsetzung im Ausland ergeben, spielt die Varianz der Vertragsrechtsordnungen ohnehin eine verhältnismäßig geringe Rolle.

ca) Die Verbraucher werden durch den gegenwärtigen Vertragsrechtspluralismus nicht wesentlich belastet. Sie können sich bei grenzüberschreitenden Geschäften 
(z.B. Internethandel) grundsätzlich auf die Geltung der Schutzstandards ihrer Heimatrechtsordnung verlassen. Das Vertrauen der Verbraucher wird durch den Entwurf nicht gestärkt, vielmehr entsteht neue Verunsicherung.

cb) Für Unternehmer, insbesondere kleine und mittlere Unternehmen (KMU), ist der Vertragsrechtspluralismus in Europa zwar u.U. ein Kostenfaktor im Binnenmarkt. Das Europäische Kaufrecht bietet für diesen Kostenfaktor aber keine Abhilfe. Denn es ist zu erwarten, dass die Kosten, die ein ,neues Europäisches Kaufvertragsrecht" durch die resultierende Rechtsunsicherheit hervorruft, deutlich stärker ins Gewicht fallen.

cc) Das Schattendasein, in dem das UN-Kaufrecht vor sich hin dümpelt, bekräftigt die mangelnde Attraktivität derartiger juristischer Experimente. Im Binnenmarkt stehen bereits jetzt 28 Rechtsordnungen (inkl. UN-Kaufrecht) zur Auswahl. Einen systematischen Nutzen aus der 29. Wahlordnung mögen allenfalls unseriöse Akteure ziehen, die von vornherein mit einer Prozessverfolgung durch die Gegenseite rechnen: Sie könnten versucht sein, die mit dem neuen Kaufrecht verbundenen Unsicherheiten als eine Art Nebelkerze gegen ihre Verfolger einzusetzen.

cd) Verglichen mit den Belastungen, die sich aus der Sprachenvielfalt und den Herausforderungen einer Rechtsberatung bzw. gerichtlichen Rechtsdurchsetzung im Ausland ergeben, dürfte die Varianz der Vertragsrechtsordnungen ohnehin eine verhältnismäßig geringe Rolle spielen. Was nützt es dem deutschen Mittelständler, dass er sich auf Europäisches Kaufrecht berufen kann, wenn er dieses in finnischer Sprache, vertreten von finnischen Anwälten vor einem finnischen Gericht und nach finnischer Verfahrensordnung durchsetzen muss?

d) Beeinträchtigung des Verbraucherschutzes: Wegfall des Mindeststandards der Heimatrechtsordnung. Nach Art. 6 II der Rom-I-VO ist den Verbrauchern bei Geschäften im Binnenmarkt weitreichend der (vertraute) Schutzstandard ihrer Heimatrechtsordnung garantiert. Der Entwurf steht in einem unauflöslichen Wertungswiderspruch zu dieser Schutzgewährleistung.

da) Gem. Art. 6 II der Rom-I-VO ist den Verbrauchern bei Binnenmarkttransaktionen weitgehend der (vertraute) Verbraucherschutzstandard ihrer Heimatrechtsordnung garantiert. Die Grundkonzeption des Entwurfs steht mit dieser Wertung in einem unauflöslichen Widerspruch: Dem Verbraucher wird aufgrund der - für ihn äußerst schwer durchschaubaren - Entscheidung für das Europäische Vertragsrecht ein Verzicht auf den Verbraucherschutzstandard seiner Heimatrechtsordnung zugemutet. 
db) Der Entwurf rechtfertigt die Zulassung einer Disposition über die Mindeststandards des nationalen Rechts damit, dass das Europäische Kaufrecht als ein Bestandteil des innerstaatlichen Rechts qualifiziert wird; es handele sich nicht um eine Rechtswahl im Sinne der Kollisionsnormen (Erwägungsgrund 10). Diese Argumentation verdeckt den wertungsmäßigen Widerspruch, da das Europäische Kaufrecht für den Verbraucher eine ebenso fremde Materie wie ausländisches Recht darstellt. Die Undurchsichtigkeit für den Verbraucher wird durch die besonderen Rechtssicherheitsprobleme des Europäischen Kaufrechts sogar noch verschärft.

e) Keine Neutralisierung der Einwände durch die optionale Ausgestaltung („Unschädlichkeits-Hypothese“). Das Wahlrecht der Vertragsparteien neutralisiert die Einwände gegen den Entwurf nicht: Ein optionales Instrument verleiht den unausgereiften akademischen Regeln Autorität und führt zu voreiligen Festlegungen im Hinblick auf spätere verbindliche Vereinheitlichungsmaßnahmen. Eine rationale Entscheidung der Parteien über die Qualität des Entwurfs ist nicht möglich, weil sich dessen konkrete Auswirkungen nicht hinreichend klar bestimmen lassen: Ohne die Ausdifferenzierungen des Richterrechts entscheiden sich die Parteien für die „Katze im Sack“.

ea) Es ist bezeichnend, dass vielfach auf die „Unschädlichkeit“ einer europäischen Wahlordnung verwiesen wird. „Unschädlichkeit“ ist kein geeigneter Maßstab für Gesetzgebung, zumal damit jedes beliebige optionale Regelwerk legitimiert werden kann.

eb) Außerdem ist die These von der Unschädlichkeit der optionalen Kaufrechtsordnung unzutreffend: Ein optionales Instrument verleiht den unausgereiften akademischen Regeln Autorität und führt zu voreiligen Festlegungen im Hinblick auf spätere verbindliche Vereinheitlichungsmaßnahmen. Auch trägt eine zusätzliche Wahlrechtsordnung zu weiterer Zersplitterung und Komplizierung des Privatrechts im europäischen Kontext bei.

ec) Im Lichte der beschriebenen Rechtsunsicherheiten (vgl. I und II) können die Parteien oder der Markt keine kalkulierbare Rechtswahl treffen. Im besten Fall hat die Entscheidung für die Einbeziehung des Europäischen Kaufrechts Zufallscharakter, im schlechtesten Fall erstrebt die stärkere Partei mit dem Verlangen nach Einbeziehung des optionalen Instruments die Herstellung eines möglichst großen Maßes an Rechtsunsicherheit, um dadurch den Spielraum für opportunistisches Verhalten zu vergrößern. 
ed) Derart unausgereifte Regelwerke sind ferner auch deswegen politisch schädlich, weil sie das Vertrauen der Bürger in die Qualität europäischer Gesetzgebung unnötig erschüttern.

f) Kompetenzdefizit. Es ist weitgehend anerkannt, dass es für ein Vertragsgesetzbuch keine belastbare EU-Kompetenz gibt. Die optionale Ausgestaltung des Europäischen Kaufrechts behebt dieses Kompetenzdefizit ebenso wenig wie die Beschränkung des Anwendungsbereichs auf Binnenmarkt-Transaktionen: Denn im Ergebnis bleibt es dabei, dass durch das Europäische Kaufrecht mitgliedstaatliches Recht außer Kraft gesetzt wird. Es ist - auch angesichts der allgemeinen Probleme einer Rechtsdurchsetzung im Ausland - nicht plausibel, dass der Binnenmarkt durch den Entwurf eines Europäischen Kaufrechts in spürbarer Weise gefördert wird (Art. 114 AEUV). Das optionale Instrument würde vielmehr der schleichenden EU-Kompetenzanmaßung weiteren Vorschub leisten.

fa) Für das Vertragsrecht im Allgemeinen und das Kaufrecht im Besonderen ist der EU im AEUV keine besondere Rechtsetzungskompetenz zugewiesen.

$\mathrm{fb})$ Es ist weitgehend anerkannt, dass die Querschnittskompetenz des Art. 114 AEUV keine belastbare Kompetenzgrundlage für ein verbindliches Vertragsrechtsgesetzbuch hergibt. Das Vertragsrecht gehört zum unverzichtbaren Kernbereich einer freiheitlichen Gesellschaftsordnung und es ist daher selbstverständlicher Bestandteil der von den Unionsverträgen respektierten Vielfalt. Bereits das Prinzip der begrenzten Einzelermächtigung steht daher breitflächigen Angleichungsmaßnahmen auf EU-Ebene entgegen.

fc) Angleichungsmaßnahmen im Bereich des Vertragsrechts kommen daher nur hinsichtlich solcher Regelungen in Betracht, deren Verschiedenheit den Binnenmarkt in besonderem Maße belastet. Der Pluralismus der Vertragsrechtsordnungen und die damit notwendig verbundenen Kosten allein sind kein legitimer Grund für Rechtssetzung durch die EU.

fd) Die Notwendigkeit eines solchen, besonders gewichtigen Binnenmarktbezugs schließt die Angleichung dispositiven Vertragsrechts grundsätzlich aus, weil hier etwaige Anpassungen von den Parteien zu erwarten sind bzw. dem Markt überlassen werden können.

fe) Hinsichtlich zwingenden Rechts und namentlich im Verbraucherschutzrecht bedarf die besondere Förderung des Binnenmarkts näherer Substantiierung (EuGH-Urteil zur Tabakrichtlinie). Hierfür genügen die pauschalen Behauptungen der EU-Kommission nicht. Plausibel ist vielmehr allein die Annahme, dass ein Europäisches Kaufrecht den Binnenmarkt überhaupt nicht beeinflussen wird. 
Dafür sprechen die offenkundigen Rechtssicherheitsprobleme, die ein neues und lückenhaftes Europäisches Kaufrecht mit sich bringt. Auch die Erfahrungen mit dem UN-Kaufrecht geben keinen Anlass zu der Erwartung, dass der Entwurf eine spürbare Marktbelebung herbeiführen kann.

ff) Der optionale Charakter des Entwurfs gleicht das Kompetenzdefizit nicht aus. Die Berufung auf Art. 114 AEUV ist bei einem optionalen Instrument schon deswegen zweifelhaft, weil der EuGH in seiner Entscheidung zur Verordnung über die Europäische Genossenschaft die Angleichungswirkung einer Wahlordnung abgelehnt und insoweit auf Art. 352 AEUV (s.u.) verwiesen hat. Allerdings bezieht sich diese Entscheidung auf den Sonderfall der Einführung einer zusätzlichen Rechtsform; auch ist die Begründung des erwähnten EuGH-Urteils deswegen paradox, weil dieses dazu führt, dass ein geringeres Maß an Verbindlichkeit (Wahlordnung) mit höheren Verfahrensschranken (Einstimmigkeit, Art. 352 AEUV) verbunden ist.

fg) Überzeugender ist es, das optionale Kaufrecht als Angleichungsmaßnahme im Sinne von Art. 114 AEUV zu qualifizieren und kompetenzmäßig wie eine auf verbindliche Rechtsgeltung gerichtete Kodifikation zu bewerten. Die Wahlmöglichkeit der Parteien ändert nichts daran, dass im Ergebnis mitgliedstaatliches Recht außer Kraft gesetzt und die Kompetenz der Mitgliedstaaten zurückgedrängt wird. Wie bei einem nicht-optionalen Kaufgesetzbuch ist daher festzustellen, dass der nach Art. 114 AEUV gebotene Binnenmarktbezug fehlt, weil das DCESL nicht dazu geeignet ist, wesentliche Behinderungen des Binnenmarkts zu beseitigen (s. Nr. 2-5).

fh) Die Beschränkung des Europäischen Kaufrechts auf Binnenmarkttransaktionen begründet ebenfalls keine Rechtssetzungskompetenz nach Art. 114 AEUV. Denn der Geltungsanspruch der mitgliedstaatlichen Rechtsordnungen bezieht sich grundsätzlich auch auf zwischenstaatliche Geschäfte - wie gerade auch die europarechtlichen Vorgaben für das Kollisionsrecht zeigen.

fi) Auch Art. 352 AEUV kommt als Kompetenzgrundlage nicht in Betracht. Diese offen formulierte Zuständigkeitsvorschrift ist im Lichte des Prinzips der begrenzten Einzelermächtigung und nach der unzweideutigen Rechtsprechung des BVerfG (Maastricht-Urteil) eng auszulegen. Die Regulierung eines so elementaren und breitflächigen Gebiets wie des Kaufrechts durch die EU würde das Prinzip der begrenzten Einzelermächtigung weitgehend aushöhlen und auf eine verdeckte Vertragsänderung hinauslaufen. 
Hans Christoph Grigoleit Stellungnahme zum Vorschlag für ein Gemeinsames Europäisches Kaufrecht

fj) Ist das Europäische Kaufrecht als optionales Instrument erst einmal kompetenziell gebilligt, so wird dadurch auch der nächste Schritt, ein verbindliches Gesetzbuch, tendenziell legitimiert. Das optionale Instrument leistet daher der schleichenden EU-Kompetenzanmaßung weiteren Vorschub. 